\title{
Rivaroxaban in patients with a recent acute coronary syndrome event: integration of trial findings into clinical practice
}

This article was published in the following Dove Press journal:

Vascular Health and Risk Management

12 May 2014

Number of times this article has been viewed

\author{
Ganesh Nallur Shivu' \\ Nick Ossei-Gerning ${ }^{2}$ \\ 'Department of Cardiology, King's \\ Mill Hospital, Sutton-in-Ashfield, \\ Nottinghamshire, UK; ${ }^{2}$ Department \\ of Cardiology, University Hospital \\ of Wales, Cardiff, UK
}

\begin{abstract}
Despite significant advances in the management of acute coronary syndrome (ACS) and long-term antiplatelet therapy after an ACS event, patients continue to be at risk of further cardiovascular events. There is evidence that recurrent events are at least partly attributed to the persistent activation of the coagulation system after ACS. Various anticoagulants, including vitamin $\mathrm{K}$ antagonists (VKAs) and non-VKA oral anticoagulants, have been evaluated in patients post-ACS, in combination with antiplatelet therapy. The desired outcome would be a further reduction of recurrent cardiovascular events with low or acceptable levels of bleeding complications. Here, we provide an overview of the current clinical trial data of non-VKA oral anticoagulants, focusing on rivaroxaban in particular, for secondary prevention in patients with a recent ACS event.
\end{abstract}

Keywords: acute coronary syndrome, anticoagulants, antiplatelet therapy

\section{Introduction}

Cardiovascular disease is the most common cause of mortality in the developed world. ${ }^{1}$ The current long-term treatment includes dual antiplatelet therapy (DAPT) with aspirin, plus a P2Y12 receptor antagonist, statins, beta-blockers, and angiotensinconverting enzyme inhibitors. ${ }^{2} \mathrm{P} 2 \mathrm{Y} 12$ receptor antagonists have established an evidence base in randomized controlled trials (CURE, ${ }^{3} \mathrm{COMMIT},{ }^{4}$ TRITON TIMI $38,{ }^{5}$ TRILOGY ACS,${ }^{6}$ and PLATO $^{7}$ ). Most patients with acute coronary syndrome (ACS) also undergo revascularization ${ }^{2}$ and, despite these treatment strategies, patients are at high risk of further cardiovascular events ${ }^{7,8}$ that are at least partly contributed to by the persistent activation of the coagulation system. ${ }^{9}$ In light of this, there has been interest in investigating anticoagulant therapy in conjunction with antiplatelet therapy to improve outcomes further in patients post-ACS. However, the inherent benefit from anticoagulant therapy has to be weighed against the increased risk of bleeding, and achieving a suitable balance is key to their utility in clinical practice. Previous studies with warfarin and antiplatelets demonstrated clinical benefits that were outweighed by an increase in bleeding. ${ }^{10}$ Novel, non-vitamin $\mathrm{K}$ antagonist (VKA) oral anticoagulants have been developed in recent years, and some have been investigated for secondary prevention after ACS. In this article, we discuss key data from recent clinical trials of the non-VKA oral anticoagulants in patients with a recent ACS event, and the potential implication of these findings for future clinical practice.
Correspondence: Nick Ossei-Gerning University Hospital of Wales, Heath Park, Cardiff CFI4 4XW, UK Tel +442920743323

Email nick.ossei-gerning@wales.nhs.uk 


\section{Rationale for the use of anticoagulants in patients after an ACS event}

The pathogenesis of atherosclerosis involves the formation of lipid-laden plaques in the arterial wall. Plaque instability results in rupture, followed by platelet activation and aggregation, and heralds the beginning of atherothrombosis, which presents clinically as ACS. Inflammation plays a key role in the onset and progression of atherosclerosis. ${ }^{11}$ Various inflammatory cells such as macrophages, neutrophils, and lymphocytes are pivotal in the process of destabilization and subsequent plaque rupture. ${ }^{12}$ The coagulation system is activated in the acute phase of ACS. ${ }^{11}$ Both Factor Xa and thrombin play a key role in the coagulation cascade, leading to clot formation (Figure 1). ${ }^{13,14}$ Clot-bound thrombin remains activated and causes progression of the thrombus, whereas any systemic thrombin is inactivated by antithrombin or thrombomodulin..$^{9,15}$ However, the activation of the coagulation system persists even after the acute phase, as shown by the levels of measured prothrombin fragment and fibrinopeptide A. This may occur in patients up to 6 months following unstable angina or myocardial infarction (MI), compared with patients with stable angina or healthy volunteers. ${ }^{9}$ This can partly explain the reason for recurrent events in patients post-ACS despite being treated with DAPT (Figure 2). Also, this provides the rationale for the use of anticoagulant therapy to further reduce recurrent events. In the ESTEEM trial, ${ }^{16}$ Wallentin et al demonstrated that reducing the procoagulant status, as measured by D-dimer levels with ximelagatran, was associated with decreased risk of new ischemic events. This effect was present irrespective of whether the reduction in D-dimer occurred spontaneously or secondary to ximelagatran. ${ }^{17}$ Parenteral anticoagulants such as heparin, low molecular weight heparin, bivalirudin, and fondaparinux - are used in the acute phase post-ACS. ${ }^{2,18}$ These agents are also used periprocedurally during percutaneous coronary intervention (PCI). Although these agents have been shown to significantly improve cardiovascular outcomes in patients with ACS, it is not practical to continue long-term treatment with these agents after hospital discharge. Previous studies of warfarin combined with aspirin have shown improved efficacy outcomes that were outweighed by an unacceptable increase in bleeding events. ${ }^{10,19}$ This was further confirmed in a meta-analysis by Andreotti et $\mathrm{al},{ }^{10}$ which demonstrated that warfarin combined with aspirin, when compared with aspirin alone, further reduced the risk of major adverse events including all-cause death, non-fatal MI, and non-fatal thromboembolic stroke, if the

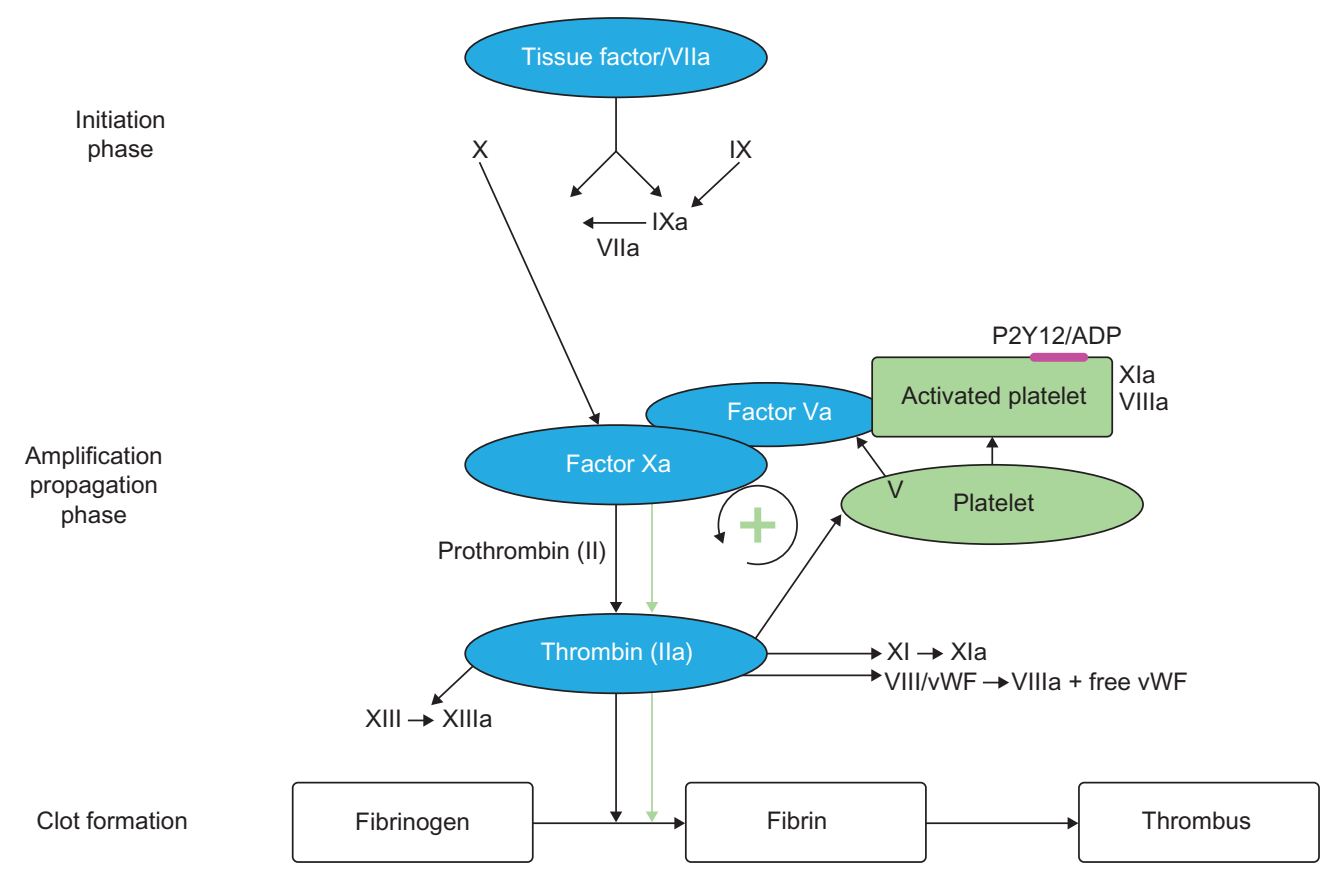

Figure I Schematic overview of the coagulation cascade.

Notes: The coagulation cascade includes three overlapping phases: initiation; amplification and propagation; and clot formation. In the initiation phase, the tissue factor/FVIla complex activates coagulation factors IX to IXa and X to Xa; FXa cleaves prothrombin, activating conversion to thrombin and generating small amounts of thrombin. In the amplification and propagation phase, thrombin signals for further platelet activation and aggregation; on the surface of the platelets, thrombin activates FV, FVIII, and FXI, and FVIIla forms a complex with FIXa, which increases thrombin generation. During the clot formation phase, thrombin catalyses the conversion of fibrinogen to fibrin, leading to thrombus formation. Data source: De Caterina et al. ${ }^{14}$

Abbreviations: ADP, adenosine diphosphate; vWF, von Willebrand factor. 


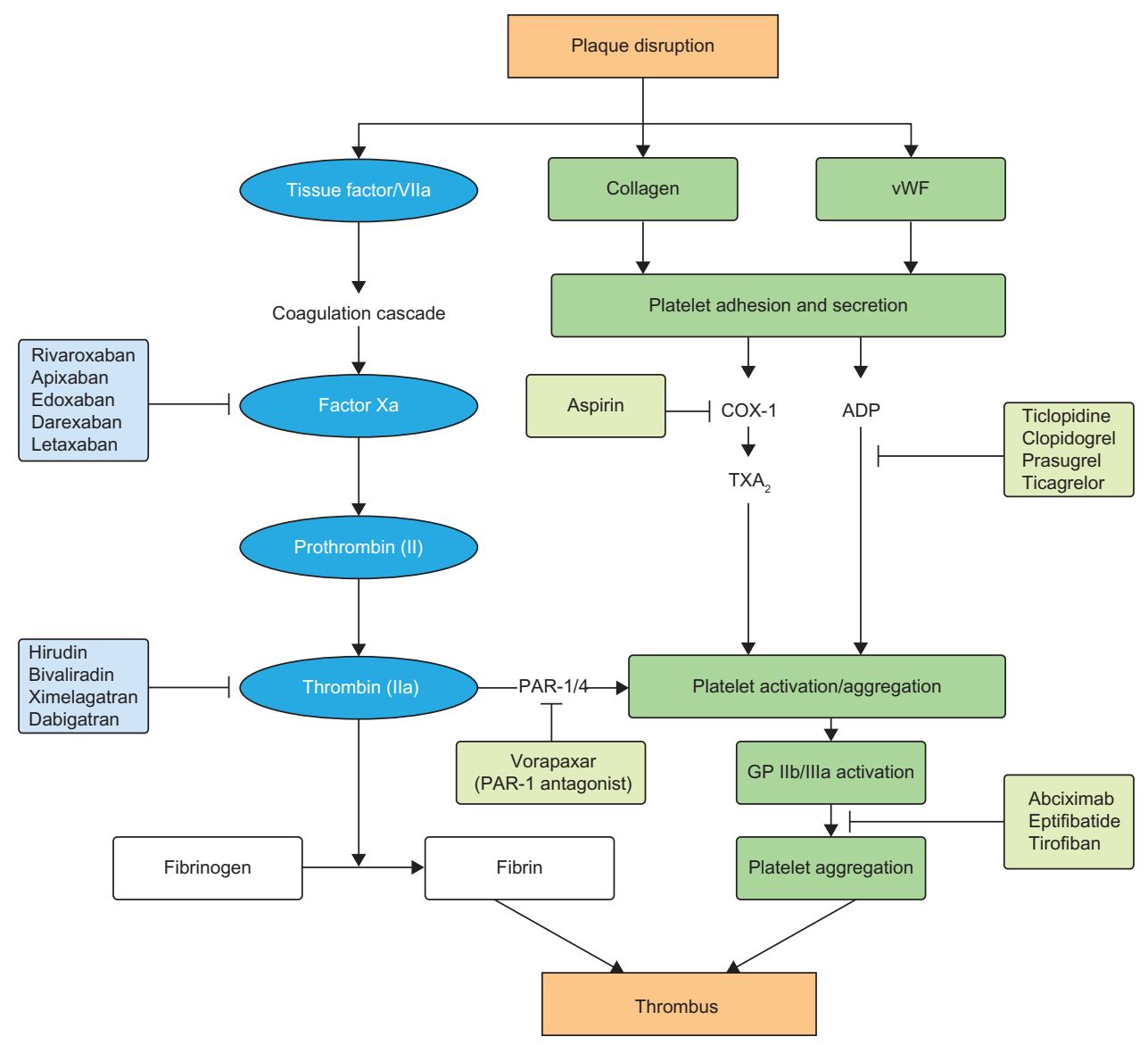

Figure 2 Pathophysiology of atherothrombosis and therapeutic targets.

Abbreviations: ADP, adenosine diphosphate; COX, cyclooxygenase; GP, glycoprotein; PAR, proteinase-activated receptor; TXA $A_{2}$, thromboxane $A_{2}$; vWF, von Willebrand factor.

international normalized ratio is $2.0-3.0$ (odds ratio: 0.73 ; 95\% confidence interval: $0.63-0.84 ; P<0.0001)$. However, the combination of warfarin and aspirin was associated with a significant increase in the risk of major bleeding. Other major drawbacks that exist with warfarin include multiple drug and food interactions, the need for regular coagulation monitoring with the international normalized ratio, and dose titration to ensure that the intensity of anticoagulation is within the therapeutic range. ${ }^{20}$

\section{Non-VKA oral anticoagulants}

The non-VKA oral anticoagulants (ie, direct Factor Xa or direct thrombin inhibitors) have several advantages over VKAs, including predictable pharmacokinetic and pharmacodynamic profiles, rapid onset and offset of action, no requirement for routine coagulation monitoring, and no clinically relevant food interactions. ${ }^{21}$ Some of these agents, such as the direct Factor Xa inhibitors rivaroxaban and apixaban, and the direct thrombin inhibitor dabigatran etexilate, have been approved for clinical use in several thromboembolic indications. ${ }^{22}$ The potential of some of these novel oral agents for secondary prevention post-ACS has also been investigated (Table 1), ${ }^{10,16,23-29}$ and the results of these studies are discussed in the following section.

\section{Direct thrombin inhibitors Ximelagatran}

Ximelagatran was the first oral direct thrombin inhibitor extensively studied as an alternative to warfarin in patients with atrial fibrillation (AF). Its potential for use in patients with ACS was studied in the ESTEEM trial ${ }^{16}$ in patients after ST-elevation or non-ST-elevation MI (STEMI or NSTEMI). Ximelagatran showed promise in reducing the risk of the composite primary endpoint of death from any cause, MI, or severe recurrent ischemic events without increase in major bleeding. However, its potential was short-lived and the drug was subsequently withdrawn from the market due to hepatotoxicity, ${ }^{16}$ but the study demonstrated the potential for oral anticoagulants in secondary prevention following ACS. 


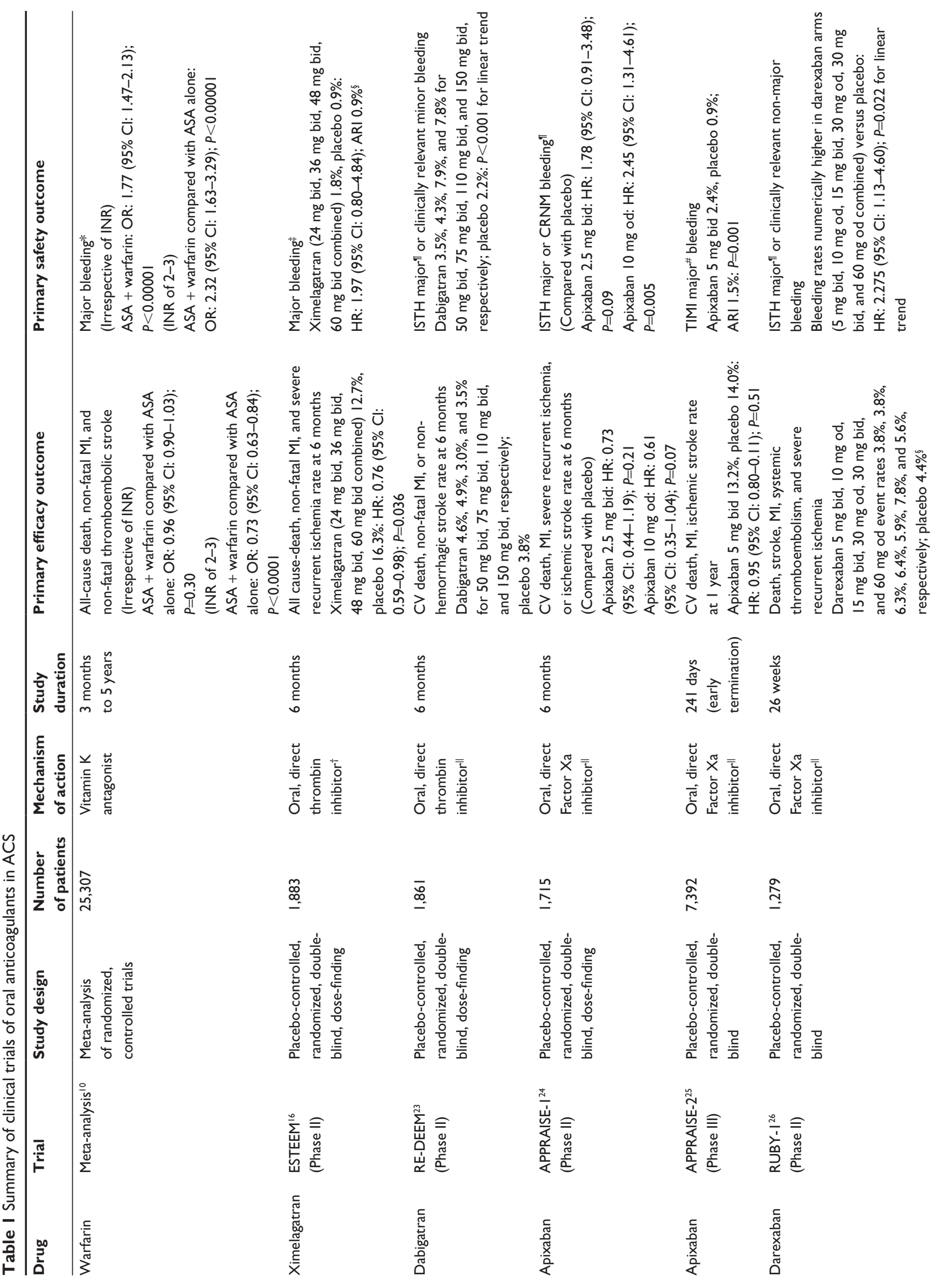



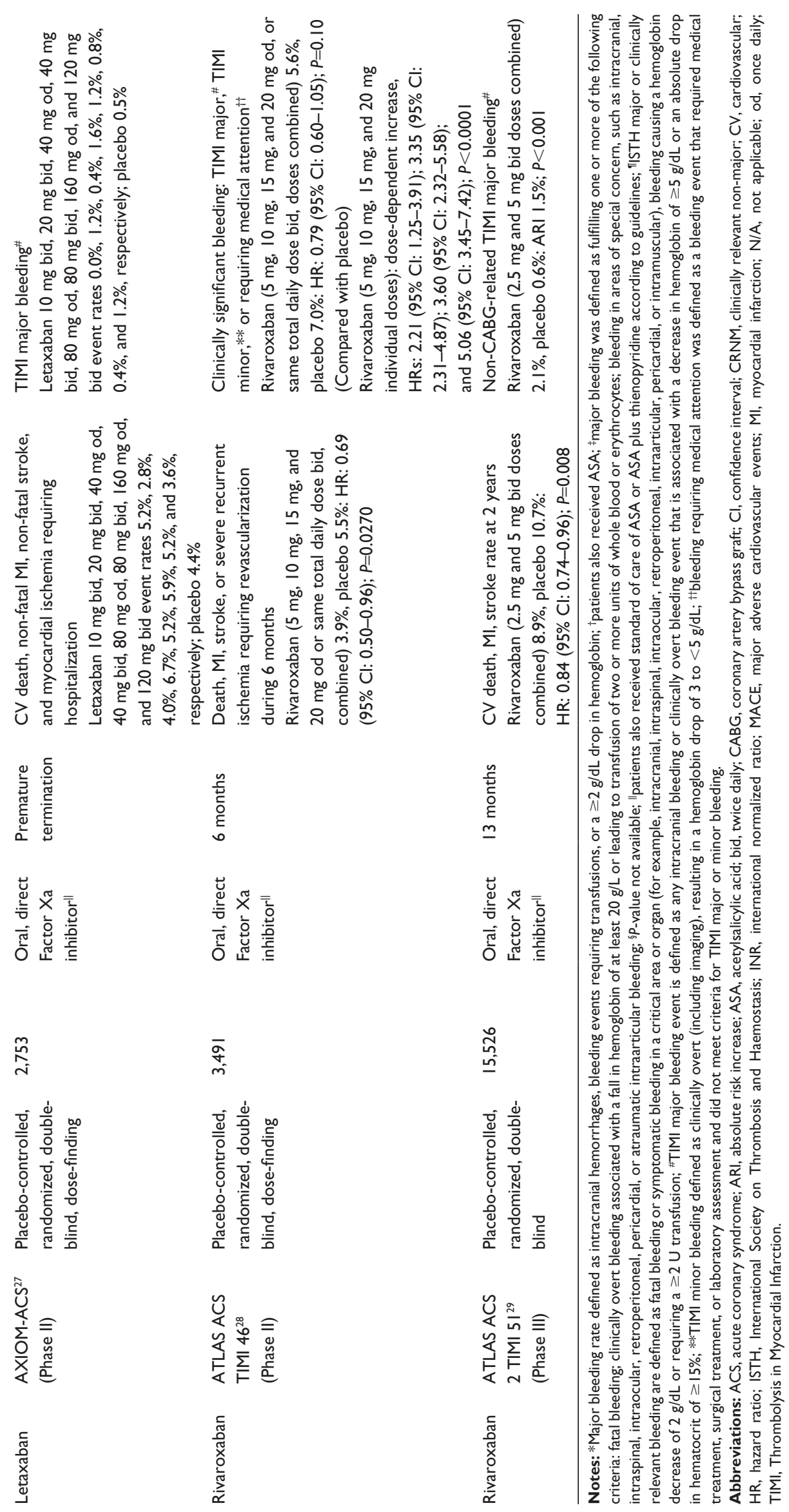


\section{Dabigatran etexilate}

The RE-DEEM trial ${ }^{23}$ was a Phase II dose-exploration study of dabigatran in patients after an ACS event. The primary endpoint of this study was the composite of major or clinically relevant non-major bleeding. Dabigatran demonstrated a dose-dependent increase in the risk of bleeding without any effect on cardiovascular ischemic endpoints (a composite of cardiovascular death, non-fatal MI, or non-hemorrhagic stroke), ${ }^{23}$ and no Phase III study has been conducted in this indication. A recent meta-analysis of trials involving oral direct thrombin inhibitors has demonstrated an increased risk of MI with these drugs compared with warfarin, with an odds ratio for dabigatran in four trials of 1.41 (95\% confidence interval: $1.09-1.83 ; P=0.009){ }^{30}$

\section{Direct Factor $\mathbf{X a}$ inhibitors Apixaban}

The APPRAISE-1 trial $^{24}$ was a Phase II, dose-exploration study conducted in patients after an ACS event, which compared increasing doses of apixaban (5-20 mg, total daily dose) and placebo plus standard antiplatelet therapy for 6 months. The study demonstrated a dose-related increase in clinically significant bleeding in patients receiving apixaban compared with placebo, although there was a trend towards a reduction in the incidence of cardiovascular events in the apixaban arm. ${ }^{24}$ Encouraged by the results of this Phase II trial, APPRAISE- ${ }^{25}$ was conducted in patients after an ACS event over 2 years. However, this Phase III study was terminated prematurely because of an increase in major bleeding events with apixaban, without a concomitant reduction in recurrent ischemic events. At termination, the composite primary endpoint of death from cardiovascular causes, MI, or ischemic stroke occurred in $7.5 \%$ of apixaban patients and in $7.9 \%$ of placebo patients; this was not statistically significant (hazard ratio [HR]: $0.95 ; P=0.51$ ). The primary safety endpoint of Thrombolysis in Myocardial Infarction (TIMI) major bleeding was statistically increased with apixaban compared with placebo (1.3\% versus $0.5 \%$; HR: 2.59; $P=0.001)$. Apixaban also increased intracranial and fatal bleeding compared with placebo. ${ }^{25}$

\section{Darexaban}

RUBY- $1^{26}$ was a Phase II exploratory trial in patients with a recent ACS event. The study assessed six darexaban dosing regimens (10-60 mg, total daily dose) compared with placebo plus standard antiplatelet therapy for 26 weeks. ${ }^{26}$ The primary safety outcome of major or clinically relevant non-major bleeding, as per the modified version of the International
Society on Thrombosis and Haemostasis's definition was numerically higher in all darexaban arms than in the placebo arm, with HRs ranging from 1.8 to $3.8 .{ }^{26}$ The composite efficacy endpoint of death from any cause, MI, stroke, or severe recurrent ischemia was numerically higher in the darexaban arms than in the placebo arm (5.6\% versus $4.4 \%) .{ }^{26}$ To date, no Phase III study has been conducted in this indication.

\section{Letaxaban}

AXIOM-ACS ${ }^{27}$ was a Phase II trial of patients following an ACS event, which included three stages of increasing doses of letaxaban. At stage 1, patients received letaxaban (10 mg, $20 \mathrm{mg}$, or $40 \mathrm{mg}$ twice daily [bid]) or placebo for 1.5-7 days; stages 2 and 3 consisted of patients receiving letaxaban doses from $40 \mathrm{mg}$ bid to $120 \mathrm{mg}$ bid. The study was terminated prematurely by the data safety monitoring board because of an increase in adverse events resulting in drug discontinuation. ${ }^{31}$ The overall rate of TIMI major bleeding was not increased with letaxaban, although a dose-dependent increase in bleeding incidence was noted. ${ }^{27}$

\section{Rivaroxaban}

Rivaroxaban is an oral, direct Factor Xa inhibitor. It has a high oral bioavailability $(80 \%-100 \%)$ and a rapid onset of action, with a half-life of 5-9 hours in healthy young individuals and 11-13 hours in the elderly. ${ }^{32-34}$ Rivaroxaban has been studied extensively for the prevention and treatment of thromboembolism and is approved for clinical use for several indications, including secondary prevention after ACS. ${ }^{35}$

\section{ATLAS ACS TIMI 46 trial}

This was a Phase II dose-exploration study ${ }^{28}$ that investigated rivaroxaban (at total daily doses of $5 \mathrm{mg}, 10 \mathrm{mg}$, $15 \mathrm{mg}$, or $20 \mathrm{mg}$ ) plus standard antiplatelet therapy in patients stabilized after an ACS event. ${ }^{28} \mathrm{~A}$ total of 3,491 patients recruited 1-7 days after hospitalization for ACS were stratified to aspirin only (stratum 1) or aspirin and a thienopyridine (stratum 2), based on physician discretion. The risk of clinically significant bleeding (the primary safety endpoint) increased in a dose-dependent manner in patients receiving rivaroxaban: the HRs were 2.21, 3.35, 3.60 , and 5.06 for the $5 \mathrm{mg}, 10 \mathrm{mg}, 15 \mathrm{mg}$, and $20 \mathrm{mg}$ doses, respectively. The rate of the primary efficacy endpoint of death, MI, stroke, or severe recurrent ischemia requiring revascularization during 6 months was lower in the rivaroxaban group than in the placebo group (5.6 versus 7.0), but this difference did not reach statistical significance $(P=0.10){ }^{28}$ 


\section{ATLAS ACS 2 TIMI 5 I trial}

Based on the Phase II study results, this Phase III study ${ }^{29}$ investigated the potential of low doses of rivaroxaban plus antiplatelet therapy to improve cardiovascular outcomes in patients with recent ACS. The study recruited 15,526 patients within 7 days of hospitalization for ACS. Patients were stratified according to thienopyridine use and then randomized in a double-blind 1:1:1 fashion to rivaroxaban $2.5 \mathrm{mg}$ bid, rivaroxaban $5 \mathrm{mg}$ bid, or placebo plus low-dose aspirin. Among the recruited patients, 50.3\% had STEMI, 25.6\% had NSTEMI, and $24.0 \%$ had unstable angina. ${ }^{29}$

Rivaroxaban (combined data for $2.5 \mathrm{mg}$ and $5 \mathrm{mg}$ bid) significantly decreased the primary composite efficacy endpoint of death from cardiovascular causes, MI, or stroke compared with placebo (8.9\% versus $10.7 \%$; HR: $0.84 ; P=0.008) .{ }^{29}$ On analysis of the components of the primary endpoint, rivaroxaban significantly decreased the rates of death from cardiovascular causes (HR: 0.80; $P=0.04)$ and MI (HR: $0.85 ; P=0.047)$. Rivaroxaban also significantly decreased the secondary composite efficacy endpoint of death from any cause, MI, or stroke compared with placebo (HR: 0.84; $P=0.006$ ). Furthermore, rivaroxa- ban significantly reduced stent thrombosis compared with placebo $(2.3 \%$ versus $2.9 \%$; HR: $0.69 ; P=0.02)$. Both the $2.5 \mathrm{mg}$ and $5 \mathrm{mg}$ arms individually caused a statistically significant reduction in the primary efficacy endpoint compared with placebo (2.5 mg: HR: $0.84 ; P=0.02 ; 5 \mathrm{mg}$ : HR: $0.85 ; P=0.03$ ). Only the $2.5 \mathrm{mg}$ dose had a survival benefit showing significant reductions in cardiovascular death (HR: 0.66; $P=0.002$ ) and all-cause death (HR: 0.68; $P=0.002$ ) (Table 2). ${ }^{29}$ There was no reduction in cardiovascular death with the $5 \mathrm{mg}$ dose (HR: $0.94 ; P=0.63$ ).

Rivaroxaban (combined data for $2.5 \mathrm{mg}$ and $5 \mathrm{mg}$ bid) significantly increased the rates of non-coronary artery bypass graft-related TIMI major bleeding (HR: 3.96; $P<0.001)$, TIMI minor bleeding $(P=0.003)$, TIMI bleeding requiring medical attention $(P<0.001)$, and intracranial hemorrhage $(P=0.009)$, but not of fatal bleeding $(P=0.66)$, compared with placebo (Table 2 ). There were numerically fewer major bleeding events associated with $2.5 \mathrm{mg}$ than with $5 \mathrm{mg}$ ( $1.8 \%$ versus $2.4 \%$ ); the $2.5 \mathrm{mg}$ dose was associated with significantly lower incidences of fatal bleeding, TIMI minor bleeding, and TIMI bleeding requiring medical attention compared with the $5 \mathrm{mg}$ dose. This could partially

Table 2 Efficacy and safety outcomes in the Phase III ATLAS ACS 2 TIMI 5 I study*

\begin{tabular}{|c|c|c|c|c|}
\hline \multirow{2}{*}{$\frac{\text { Endpoint }}{\text { Efficacy }}$} & \multicolumn{3}{|c|}{ Rivaroxaban } & \multirow{2}{*}{$\begin{array}{l}\text { Placebo } \\
(\mathrm{N}=5,1 \mid 3)\end{array}$} \\
\hline & $\begin{array}{l}2.5 \mathrm{mg} \text { bid } \\
(\mathrm{N}=5, \mathrm{I} \mid 4)\end{array}$ & $\begin{array}{l}5 \mathrm{mg} \text { bid } \\
(\mathrm{N}=5,1 \mid 5)\end{array}$ & $\begin{array}{l}\text { Combined } \\
(\mathrm{N}=10,229)\end{array}$ & \\
\hline Death from cardiovascular causes, & $313(9.1)$ & $313(8.8)$ & $626(8.9)$ & $376(10.7)$ \\
\hline \multicolumn{5}{|l|}{ MI, or stroke - primary endpoint } \\
\hline Death from cardiovascular causes & $94(2.7)$ & $132(4.0)$ & $226(3.3)$ & $143(4.1)$ \\
\hline MI & $205(6.1)$ & $179(4.9)$ & $384(5.5)$ & $229(6.6)$ \\
\hline \multicolumn{5}{|l|}{ Stroke } \\
\hline Any & $46(1.4)$ & $54(1.7)$ & $100(1.6)$ & $4 I(I .2)$ \\
\hline Ischemic & $30(1.0)$ & $35(0.9)$ & $65(0.9)$ & $34(1.0)$ \\
\hline $\begin{array}{l}\text { Death from any cause, } \mathrm{MI} \text {, or stroke - } \\
\text { secondary endpoint }\end{array}$ & $320(9.3)$ & $321(9.1)$ & $64 \mid(9.2)$ & $386(11.0)$ \\
\hline Death from any cause & $103(2.9)$ & $142(4.4)$ & $245(3.7)$ & $153(4.5)$ \\
\hline Stent thrombosis & $47(2.2)$ & $5 I(2.3)$ & $98(2.3)$ & $72(2.9)$ \\
\hline Safety & $(\mathrm{N}=5,115)$ & $(\mathrm{N}=5, I \mid 0)$ & $(N=10,225)$ & $(\mathrm{N}=5,125)$ \\
\hline TIMI major bleeding not associated with CABG & $65(1.8)$ & $82(2.4)$ & $147(2.1)$ & $19(0.6)$ \\
\hline TIMI minor bleeding & $32(0.9)$ & $49(1.6)$ & $81(1.3)$ & $20(0.5)$ \\
\hline TIMI bleeding requiring medical attention & $492(12.9)$ & $637(16.2)$ & $1,129(14.5)$ & $282(7.5)$ \\
\hline Intracranial hemorrhage & $14(0.4)$ & $18(0.7)$ & $32(0.6)$ & $5(0.2)$ \\
\hline Fatal bleeding & $6(0.1)$ & $15(0.4)$ & $21(0.3)$ & $9(0.2)$ \\
\hline
\end{tabular}

Notes: *Event rates are reported as Kaplan-Meier estimates through 24 months, and are thus not presented as numerical percentages. Data for the efficacy endpoints correspond to the mITT analysis, with P-values presented for both mITT and ITT analyses. Before the unblinding of the study results, 184 patients were excluded from the efficacy analysis because of violations in the Good Clinical Practice guidelines at three sites. MI and stroke categories include fatal and non-fatal events. Stroke includes ischemic, hemorrhagic, and stroke of uncertain cause. Stent thrombosis (definite, probable, or possible) analyses were conducted among patients who had received a stent prior to randomization. Data for the safety endpoints correspond to the safety analysis. From The New England Journal of Medicine, Mega JL, Braunwald E, Wiviott SD, et al, ATLAS ACS 2-TIMI 5I Investigators, Rivaroxaban in patients with a recent acute coronary syndrome, Volume 366, Pages 9-19.29 Copyright (C) 20I2 Massachusetts Medical Society. Reprinted with permission from Massachusetts Medical Society.

Abbreviations: bid, twice daily; CABG, coronary artery bypass graft; ITT, intention-to-treat; MI, myocardial infarction; mITT, modified intention-to-treat; TIMI, Thrombolysis in Myocardial Infarction. 
explain the reduction in mortality seen with the $2.5 \mathrm{mg}$ dose, but not with the $5 \mathrm{mg}$ dose (Table 2). ${ }^{29,36}$

\section{Rivaroxaban in patients with ACS: what are the key factors pertaining to its use in daily clinical practice?}

Use of anticoagulation is beneficial in reducing major adverse cardiovascular events after an initial ACS event, but these benefits can be negated by the increased risk of major bleeding. ${ }^{10}$ Rivaroxaban is the first oral anticoagulant to show improved outcomes in ACS without increasing fatal bleeding, ${ }^{29}$ if used in combination with standard antiplatelet therapy, finding the "sweet spot" in providing net clinical benefit. There are several factors that may have contributed to the different observed outcomes between the non-VKA oral anticoagulants, such as the failure of apixaban to show a beneficial effect. The ATLAS ACS 2 TIMI 51 trial used much lower doses of rivaroxaban ( $2.5 \mathrm{mg}$ and $5 \mathrm{mg}$ bid) than the doses used for other indications; the trial for stroke prevention in patients with non-valvular $\mathrm{AF}$, for instance, used a dose of $20 \mathrm{mg}$ once daily (od). ${ }^{37} \mathrm{In}$ contrast, APPRAISE- $2^{25}$ used a similar dose of apixaban to that used in the stroke prevention study, ${ }^{38}$ which may explain the increased risk of major bleeding including intracranial and fatal bleeding events. It seems clear that using high doses in combination with antiplatelets was the major contributor for the failure of the other non-VKA oral anticoagulants in ACS trials. Furthermore, ATLAS ACS 2 TIMI 51 ${ }^{29}$ excluded patients who were at high risk of bleeding, such as patients with platelet counts $<90,000 / \mathrm{mm}^{3}$, hemoglobin $<10 \mathrm{~g} / \mathrm{dL}$, creatinine clearance $<30 \mathrm{~mL} /$ minute, significant gastrointestinal bleeding in the past 12 months, previous intracranial hemorrhage, or previous transient ischemic attack (TIA)/ stroke. The APPRAISE-2 study, ${ }^{25}$ however, included patients who were at a high bleeding risk and had cerebrovascular disease, peripheral vascular disease, or renal insufficiency, and patients with heart failure. Data from the TRITON-TIMI $38^{5}$ study also showed a significant increase in major bleeding with no efficacy benefit for prasugrel compared with clopidogrel in patients with previous stroke or TIA. Furthermore, the mean age group for the ATLAS ACS 2 TIMI $51^{29}$ study was 62 years, with approximately $9 \%$ of patients aged $>75$ years; the median age for the APPRAISE- ${ }^{25}$ study was 67 years, with nearly $60 \%$ of patients aged $>65$ years. This may have been another factor contributing to the increased bleeding complications noted in this study. However, it is important to remember that, compared with antiplatelet therapy alone, rivaroxaban increased the risk of TIMI major bleeding, TIMI minor bleeding, TIMI bleeding requiring medical attention, and intracranial bleeding; therefore, bleeding risk should be carefully considered before using this drug in patients with ACS. ${ }^{29}$

As discussed, rivaroxaban $2.5 \mathrm{mg}$ significantly decreased death from cardiovascular or any cause - an effect that was not seen in the $5 \mathrm{mg}$ dose cohort. ${ }^{29}$ The rate of major bleeding associated with the $2.5 \mathrm{mg}$ dose was numerically lower than that associated with the $5 \mathrm{mg}$ dose (1.8\% versus $2.4 \%$ ). This is consistent with findings from other major trials in patients with ACS - such as HORIZONS AMI ${ }^{39}$ and OASIS-5 (Fifth Organization to Assess Strategies in Acute Ischemic Syndromes), ${ }^{40}$ in which decreased bleeding was consistently associated with mortality benefit - which highlights the importance of bleeding and associated mortality. The key message, therefore, is to use the low dose of rivaroxaban (2.5 mg bid) after a careful assessment of bleeding risk. Some clinicians may wish to consider this therapy in the higher ischemic risk group with a bleeding risk that is not unacceptably high. For example, because rivaroxaban has been shown to significantly reduce the rates of stent thrombosis, it should be considered in patients who undergo PCI as the revascularization strategy for their ACS and who have a high stent thrombosis risk but an acceptable bleeding risk. Rivaroxaban $2.5 \mathrm{mg}$ bid is now approved in the European Union (but not in the US) for the prevention of atherothrombotic events in adults with ACS who have elevated cardiac biomarkers, combined with aspirin with or without clopidogrel or ticlopidine. ${ }^{35}$ There are no data currently available for rivaroxaban in combination with other DAPT regimens (for example, aspirin plus prasugrel or aspirin plus ticagrelor). Therefore, rivaroxaban should not be used in combination with these newer DAPT regimens, especially because these agents caused more bleeding than aspirin plus clopidogrel in clinical studies. ${ }^{5,7}$

A further question is whether the triple therapy of rivaroxaban, aspirin, and clopidogrel is better than the newer DAPT combination of aspirin plus prasugrel or aspirin plus ticagrelor. There are some interesting observations to note in the ATLAS ACS 2 TIMI $51^{29}$ and PLATO trials. ${ }^{7}$ In the PLATO trial, ${ }^{7}$ patients were randomized in the acute phase (within 24 hours), whereas in the ATLAS trial, ${ }^{29}$ patients were randomized in the subacute phase, up to 7 days after an ACS event. The primary endpoint occurred in $11.7 \%$ of patients in the clopidogrel arm in $\mathrm{PLATO}^{7}$ and $10.7 \%$ in the placebo arm (predominantly aspirin with clopidogrel) in ATLAS ACS 2 TIMI 51. ${ }^{29}$ Interestingly, the addition of rivaroxaban $(2.5 \mathrm{mg}$ dose $)$ resulted in a $34 \%$ relative risk reduction in cardiovascular mortality in the ATLAS 
ACS 2 TIMI 51 trial, ${ }^{29}$ whereas a $20 \%$ reduction was observed with ticagrelor in the PLATO trial $^{7}$ (compared with clopidogrel).

\section{Management of bleeding events}

A concern associated with the use of non-VKA oral anticoagulants (including rivaroxaban) is the absence of a specific antidote that can be used to rapidly reverse the anticoagulation effect in case of emergency, such as life-threatening bleeding events or emergency surgery. ${ }^{41}$ Combining any anticoagulant with antiplatelet therapy will inevitably increase the risk of bleeding; therefore, careful assessment of bleeding risk (for example, the CRUSADE bleeding score ${ }^{42}$ - low red blood cell count, renal impairment, prior vascular disease, diabetes, and so on) and careful monitoring for signs or symptoms of bleeding will be required. To prevent major or fatal bleeding events, rivaroxaban administration is contraindicated in patients with clinically significant active bleeding, in patients with hepatic disease associated with clinically relevant bleeding and coagulopathy, and in patients with prior stroke or TIA. Rivaroxaban is also not recommended in patients with severe renal impairment (creatinine clearance $<15 \mathrm{~mL} /$ minute) in all of its approved indications. ${ }^{43}$

The mainstay of treatment for patients who have a major bleeding event with rivaroxaban is to stop the drug and provide supportive care while waiting for the anticoagulation effect to wane. This supportive care includes fluid or blood replacement, hemodynamic support, mechanical compression, and surgical hemostasis. ${ }^{44}$ Rivaroxaban may affect international normalized ratio measurements, but the international normalized ratio is not recommended for monitoring the anticoagulant activity or dosing of rivaroxaban. ${ }^{43}$

There is some emerging evidence that prothrombin complex concentrate could reverse the anticoagulant effects of rivaroxaban ${ }^{45,46}$ and dabigatran etexilate. ${ }^{46}$ In animal studies and Phase I studies conducted with healthy human volunteers, prothrombin complex concentrate (four-factor) infusion normalized the prolonged prothrombin time induced by rivaroxaban, and the effect was sustained for 24 hours, showing promise as an effective reversal agent for rivaroxaban. ${ }^{45,47}$ However, further studies in patients with bleeding are required to confirm its potential clinical utility. Many studies are being undertaken to find reversal agents for Factor Xa inhibitors. ${ }^{46-48}$ One such drug, PRT445, has undergone Phase I and Phase II studies, which have confirmed it has a good safety profile in healthy volunteers. ${ }^{48}$ Further studies are eagerly awaited.

\section{Other considerations for integration of rivaroxaban into clinical practice}

In clinical practice, the question of when to start rivaroxaban therapy is an important one. For example, heparin, a parenteral anticoagulant, is commonly used in the acute setting. By contrast, oral anticoagulants such as rivaroxaban have been tested in the subacute phase. Indeed, in the ATLAS ACS 2 TIMI 51 Phase III trial, ${ }^{29}$ the average number of days at which rivaroxaban treatment was initiated was 4.7 days after the index event. When using the two different anticoagulant approaches (parenteral and oral) in the acute and subacute settings, a switch from one agent to the other must be managed to ensure adequate anticoagulation is maintained. Guidance for switching from a parenteral anticoagulant to rivaroxaban advises that "rivaroxaban should be started 0 to 2 hours before the time of the next scheduled administration of the parenteral anticoagulant or at the time of discontinuation of continually administered parenteral anticoagulant"; however, guidance for switching from rivaroxaban to a parenteral anticoagulant advises that "the first dose of the parenteral anticoagulant should be given at the time the next rivaroxaban dose would be taken". 43

A common clinical scenario encountered in ACS management is the need for oral anticoagulants because of concomitant AF or mechanical heart valves. Up to $30 \%$ of patients who require long-term anticoagulation have associated ischemic heart disease. ${ }^{49}$ Clinical data on the use of rivaroxaban in patients with both ACS and AF are not available and, therefore, there are no recommendations regarding specific rivaroxaban doses to be used in these patients. The WOEST study ${ }^{49}$ is a recent open-label randomized trial, which compared triple therapy (warfarin, aspirin, and clopidogrel) with dual therapy (clopidogrel and warfarin) in approximately 600 patients who needed long-term anticoagulation and who were undergoing PCI. The primary endpoint (any bleeding events) occurred more frequently in the triple-therapy cohort compared with in the dual-therapy cohort (44.4\% versus 19.4\%, respectively; $P<0.001$ ). The rate of combined secondary endpoint of death, MI, stroke, target vessel revascularization, and stent thrombosis was statistically significantly higher in the triple-therapy cohort compared with in the dual-therapy cohort $(17.6 \%$ versus $11.1 \%$, respectively; $P=0.025)$. Dual therapy conferred a mortality benefit compared with triple therapy $(6.3 \%$ versus $2.6 \%$, respectively; $P=0.027$ ), again strengthening the argument that less bleeding is always good. This is the first study to give an inclination that aspirin may be omitted 
safely and that the combination of an oral anticoagulant and clopidogrel offers benefits in reducing the risk of bleeding without significantly increasing cardiovascular outcomes. This trial also raises the question of whether combination therapy with an anticoagulant and an antiplatelet agent is better than DAPT in all patients with ACS. Consistent with the WOEST study, ${ }^{49}$ the Phase II ATLAS ACS TIMI 46 trial $^{28}$ showed that clinically significant bleeding was lower in the rivaroxaban plus aspirin group than in the rivaroxaban plus aspirin and thienopyridine group, irrespective of whether dosing is once daily or twice daily. ${ }^{28}$ Further studies regarding drug combinations are eagerly awaited.

PIONEER AF-PCI ${ }^{50}$ is a new Phase III study that will evaluate three antithrombotic regimens after PCI, with stent placement in patients with nonvalvular AF. The treatment arms are: 1) rivaroxaban $2.5 \mathrm{mg}$ bid plus DAPT for $1 \mathrm{month}$, 6 months, or 12 months, followed by rivaroxaban $15 \mathrm{mg}$ od plus aspirin; 2) VKA treatment plus DAPT for 1 month, 6 months, or 12 months, followed by VKA plus aspirin until end of treatment (at 12 months); and 3) rivaroxaban $15 \mathrm{mg}$ od plus clopidogrel for 12 months. Which drug combinations are best for different clinical settings are only now beginning to emerge and will doubtless continue to evolve our understanding of the "sweet spot" between ischemia and bleeding.

Although clinical evidence is lacking at present, there is a growing consensus among experts that rivaroxaban can be used in combination with DAPT in patients with very diffuse coronary artery disease who do not have any revascularization options, and in patients with ectatic atherosclerotic disease and sluggish coronary flow. In the latter situation, warfarin is often given, although there is no evidence base to support this strategy.

\section{Conclusion}

Emerging evidence indicates that anticoagulant therapy combined with standard antiplatelet therapy can further reduce the risk of recurrent cardiovascular events in patients with ACS. Non-VKA oral anticoagulants have several benefits compared with VKAs in terms of pharmacodynamics; interactions with drugs, food, and alcohol; and the need for ongoing coagulation monitoring. In contrast to VKAs and other novel oral anticoagulants, the addition of low doses of rivaroxaban to DAPT reduces the risk of death from cardiovascular events without increasing fatal bleeding in patients with a recent ACS event. Long-term anticoagulation with a low dose of rivaroxaban represents a new adjunctive treatment strategy for secondary prevention in patients with ACS.
It is clear that antithrombotic therapy should be individualized for patients after an ACS event. The addition of rivaroxaban $2.5 \mathrm{mg}$ to standard antiplatelet therapy should be considered in patients with ACS who have high ischemic but low bleeding risk and perhaps also in those with AF, after an assessment of bleeding risk.

\section{Acknowledgments}

The authors would like to acknowledge Yong-Ling Liu and Abigail Macleod, who provided editorial assistance with funding from Janssen Scientific Affairs, LLC and Bayer HealthCare Pharmaceuticals.

This work was supported by Janssen Scientific Affairs, LLC, and Bayer HealthCare Pharmaceuticals (for editorial support only).

\section{Disclosure}

Dr Ossei-Gerning received consultancy fees from Bayer HealthCare and has no conflicts of interest in relation to this manuscript. Dr Nallur Shivu has no conflicts of interests to declare. The authors report no other conflicts of interest in this work.

\section{References}

1. Institute for Health Metrics and Evaluation. Global burden of disease (GBD) visualizations. Seattle, WA: Institute for Health Metrics and Evaluation; 2013. Available from: http://www.healthmetricsandevaluation.org/gbd/visualizations/country. Accessed May 15, 2013.

2. Hamm CW, Bassand JP, Agewall S, et al; ESC Committee for Practice Guidelines. ESC Guidelines for the management of acute coronary syndromes in patients presenting without persistent ST-segment elevation: The Task Force for the management of acute coronary syndromes (ACS) in patients presenting without persistent ST-segment elevation of the European Society of Cardiology (ESC). Eur Heart J. 2011;32(23):2999-3054.

3. Yusuf S, Zhao F, Mehta SR, Chrolavicius S, Tognoni G, Fox KK; Clopidogrel in Unstable Angina to Prevent Recurrent Events Trial Investigators. Effects of clopidogrel in addition to aspirin in patients with acute coronary syndromes without ST-segment elevation. $N$ Engl J Med. 2001;345(7):494-502.

4. Chen ZM, Jiang LX, Chen YP, et al; COMMIT (ClOpidogrel and Metoprolol in Myocardial Infarction Trial) collaborative group. Addition of clopidogrel to aspirin in 45,852 patients with acute myocardial infarction: randomised placebo-controlled trial. Lancet. 2005;366(9497):1607-1621.

5. Wiviott SD, Braunwald E, McCabe $\mathrm{CH}$, et al; TRITON-TIMI 38 Investigators. Prasugrel versus clopidogrel in patients with acute coronary syndromes. N Engl J Med. 2007;357(20):2001-2015.

6. Roe MT, Armstrong PW, Fox KA, et al; TRILOGY ACS Investigators. Prasugrel versus clopidogrel for acute coronary syndromes without revascularization. N Engl J Med. 2012;367(14):1297-1309.

7. Wallentin L, Becker RC, Budaj A, et al; PLATO Investigators. Ticagrelor versus clopidogrel in patients with acute coronary syndromes. $N$ Engl $J$ Med. 2009;361(11):1045-1057.

8. [No authors listed]. Collaborative overview of randomised trials of antiplatelet therapy - I: Prevention of death, myocardial infarction, and stroke by prolonged antiplatelet therapy in various categories of patients. Antiplatelet Trialists' Collaboration. BMJ. 1994;308(6921):81-106. 
9. Merlini PA, Bauer KA, Oltrona L, et al. Persistent activation of coagulation mechanism in unstable angina and myocardial infarction. Circulation. 1994;90(1):61-68.

10. Andreotti F, Testa L, Biondi-Zoccai GG, Crea F. Aspirin plus warfarin compared to aspirin alone after acute coronary syndromes: an updated and comprehensive meta-analysis of 25,307 patients. Eur Heart J. 2006;27(5):519-526.

11. Mackman N. Triggers, targets and treatments for thrombosis. Nature. 2008;451(7181):914-918.

12. Borissoff JI, Spronk HM, ten Cate H. The hemostatic system as a modulator of atherosclerosis. $N$ Engl J Med. 2011;364(18): 1746-1760.

13. Mann KG, Brummel K, Butenas $\mathrm{S}$. What is all that thrombin for? J Thromb Haemost. 2003;1(7):1504-1514.

14. De Caterina R, Husted S, Wallentin L, et al; European Society of Cardiology Working Group on Thrombosis Task Force on Anticoagulants in Heart Disease. General mechanisms of coagulation and targets of anticoagulants (Section I). Position Paper of the ESC Working Group on Thrombosis - Task Force on Anticoagulants in Heart Disease. Thromb Haemost. 2013;109(4):569-579.

15. Turpie AG, Esmon C. Venous and arterial thrombosis - pathogenesis and the rationale for anticoagulation. Thromb Haemost. 2011;105(4): 586-596.

16. Wallentin L, Wilcox RG, Weaver WD, et al; ESTEEM Investigators Oral ximelagatran for secondary prophylaxis after myocardial infarction: the ESTEEM randomised controlled trial. Lancet. 2003;362(9386): 789-797.

17. Christersson C, Oldgren J, Bylock A, Siegbahn A, Wallentin L. Early decrease in coagulation activity after myocardial infarction is associated with lower risk of new ischaemic events: observations from the ESTEEM Trial. Eur Heart J. 2007;28(6):692-698.

18. Steg PG, James SK, Atar D, et al; Task Force on the management of ST-segment elevation acute myocardial infarction of the European Society of Cardiology (ESC). ESC Guidelines for the management of acute myocardial infarction in patients presenting with ST-segment elevation. Eur Heart J. 2012;33(20):2569-2619.

19. Testa L, Zoccai GB, Porto I, et al. Adjusted indirect meta-analysis of aspirin plus warfarin at international normalized ratios 2 to 3 versus aspirin plus clopidogrel after acute coronary syndromes. Am J Cardiol. 2007;99(12):1637-1642.

20. Ansell J, Hirsh J, Hylek E, Jacobson A, Crowther M, Palareti G; American College of Chest Physicians. Pharmacology and management of the vitamin K antagonists: American College of Chest Physicians Evidence-Based Clinical Practice Guidelines (8th Edition). Chest. 2008;133(6 Suppl):160S-198S.

21. Eriksson BI, Quinlan DJ, Eikelboom JW. Novel oral factor Xa and thrombin inhibitors in the management of thromboembolism. Annu Rev Med. 2011;62:41-57.

22. Wanat MA. Novel oral anticoagulants: a review of new agents. Postgrad Med. 2013;125(4):103-114.

23. Oldgren J, Budaj A, Granger CB, et al; RE-DEEM Investigators. Dabigatran vs. placebo in patients with acute coronary syndromes on dual antiplatelet therapy: a randomized, double-blind, phase II trial. Eur Heart J. 2011;32(22):2781-2789.

24. Alexander JH, Becker RC, Bhatt DL, et al; APPRAISE Steering Committee and Investigators. Apixaban, an oral, direct, selective factor Xa inhibitor, in combination with antiplatelet therapy after acute coronary syndrome: results of the Apixaban for Prevention of Acute Ischemic and Safety Events (APPRAISE) trial. Circulation. 2009;119(22): 2877-2885.

25. Alexander JH, Lopes RD, James S, et al; APPRAISE-2 Investigators. Apixaban with antiplatelet therapy after acute coronary syndrome. N Engl J Med. 2011;365(8):699-708.

26. Steg PG, Mehta SR, Jukema JW, et al; RUBY-1 Investigators. RUBY-1: a randomized, double-blind, placebo-controlled trial of the safety and tolerability of the novel oral factor Xa inhibitor darexaban (YM150) following acute coronary syndrome. Eur Heart J. 2011;32(20): 2541-2554.
27. Goldstein S, Bates E, Bhatt D, et al. Safety evaluation of the factor Xa inhibitor TAK-442 in subjects with acute coronary syndromes: phase 2 AXIOM-ACS trial results. Eur Heart J. 2011;32(Abstract Supplement): Abstract P2430.

28. Mega JL, Braunwald E, Mohanavelu S, et al; ATLAS ACS-TIMI 46 study group. Rivaroxaban versus placebo in patients with acute coronary syndromes (ATLAS ACS-TIMI 46): a randomised, double-blind, phase II trial. Lancet. 2009;374(9683):29-38.

29. Mega JL, Braunwald E, Wiviott SD, et al; ATLAS ACS 2-TIMI 51 Investigators. Rivaroxaban in patients with a recent acute coronary syndrome. N Engl J Med. 2012;366(1):9-19.

30. Artang R, Rome E, Nielsen JD, Vidaillet HJ. Meta-analysis of randomized controlled trials on risk of myocardial infarction from the use of oral direct thrombin inhibitors. Am J Cardiol. 2013;112(12): 1973-1979.

31. Ahrens I, Peter K, Lip GY, Bode C. Development and clinical applications of novel oral anticoagulants. Part II. Drugs under clinical investigation. Discov Med. 2012;13(73):445-450.

32. Kubitza D, Becka M, Wensing G, Voith B, Zuehlsdorf M. Safety, pharmacodynamics, and pharmacokinetics of BAY 59-7939 - an oral, direct Factor Xa inhibitor - after multiple dosing in healthy male subjects. Eur J Clin Pharmacol. 2005;61(12):873-880.

33. Kubitza D, Becka M, Roth A, Mueck W. Dose-escalation study of the pharmacokinetics and pharmacodynamics of rivaroxaban in healthy elderly subjects. Curr Med Res Opin. 2008;24(10):2757-2765.

34. Perzborn E, Roehrig S, Straub A, Kubitza D, Misselwitz F. The discovery and development of rivaroxaban, an oral, direct factor Xa inhibitor. Nat Rev Drug Discov. 2011;10(1):61-75.

35. Committee for Medicinal Products for Human Use (CHMP). Summary of Opinion (Post Authorisation): Xarelto, rivaroxaban. London, UK: European Medicines Agency; 2013. Available from: http://www. ema.europa.eu/docs/en_GB/document_library/Summary_of_opinion/ human/000944/WC500140679.pdf. Accessed July 16, 2013.

36. Mega JL, Braunwald E, Wiviott SD, et al. Comparison of the efficacy and safety of two rivaroxaban doses in acute coronary syndrome (from ATLAS ACS 2-TIMI 51). Am J Cardiol. 2013;112(4):472-478.

37. Patel MR, Mahaffey KW, Garg J, et al; ROCKET AF Investigators. Rivaroxaban versus warfarin in nonvalvular atrial fibrillation. $N$ Engl J Med. 2011;365(10):883-891.

38. Granger CB, Alexander JH, McMurray JJ, et al; ARISTOTLE Committees and Investigators. Apixaban versus warfarin in patients with atrial fibrillation. N Engl J Med. 2011;365(11):981-992.

39. Rakowski T, Dudek D, Dziewierz A, et al. Impact of infarct-related artery patency before primary PCI on outcome in patients with STsegment elevation myocardial infarction: the HORIZONS-AMI trial. EuroIntervention. 2013;8(11):1307-1314.

40. Sculpher MJ, Lozano-Ortega G, Sambrook J, et al. Fondaparinux versus Enoxaparin in non-ST-elevation acute coronary syndromes: short-term cost and long-term cost-effectiveness using data from the Fifth Organization to Assess Strategies in Acute Ischemic Syndromes Investigators (OASIS-5) trial. Am Heart J. 2009;157(5):845-852.

41. Turpie AG, Kreutz R, Llau J, Norrving B, Haas S. Management consensus guidance for the use of rivaroxaban - an oral, direct factor Xa inhibitor. Thromb Haemost. 2012;108(5):876-886.

42. Mehran R, Pocock SJ, Nikolsky E, et al. A risk score to predict bleeding in patients with acute coronary syndromes. $\mathrm{J}$ Am Coll Cardiol. 2010;55(23):2556-2566.

43. Bayer Pharma AG. Xarelto ${ }^{\circledR}$ Summary of Product Characteristics $-E U$. Berlin, Germany: Bayer Pharma AG; 2013. Available from: http:// www.xarelto.com/html/downloads/2013-05_XRL_SPC_final_low.pdf. Accessed June 11, 2013.

44. Peacock WF, Gearhart MM, Mills RM. Emergency management of bleeding associated with old and new oral anticoagulants. Clin Cardiol. 2012;35(12):730-737.

45. Eerenberg ES, Kamphuisen PW, Sijpkens MK, Meijers JC, Buller HR, Levi M. Reversal of rivaroxaban and dabigatran by prothrombin complex concentrate: a randomized, placebo-controlled, crossover study in healthy subjects. Circulation. 2011;124(14):1573-1579. 
46. Marlu R, Hodaj E, Paris A, Albaladejo P, Cracowski JL, Pernod G. Effect of non-specific reversal agents on anticoagulant activity of dabigatran and rivaroxaban: a randomised crossover ex vivo study in healthy volunteers. Thromb Haemost. 2012;108(2):217-224.

47. Perzborn E, Gruber A, Tinel H, et al. Reversal of rivaroxaban anticoagulation by haemostatic agents in rats and primates. Thromb Haemost. 2013;110(1):162-172.

48. Mark C, Vandana M, Michael K, et al. A phase 2 randomized, double-blind, placebo-controlled trial demonstrating reversal of rivaroxaban-induced anticoagulation in healthy subjects by andexanet alfa (PRT064445), an antidote for Fxa inhibitors [abstract]. Blood. 2013;122(21):3636.
49. Dewilde WJ, Oirbans T, Verheugt FW, et al; WOEST study investigators. Use of clopidogrel with or without aspirin in patients taking oral anticoagulant therapy and undergoing percutaneous coronary intervention: an open-label, randomised, controlled trial. Lancet. 2013;381(9872):1107-1115.

50. Bayer Pharma AG. A study exploring two strategies of rivaroxaban (JNJ39039039; BAY-59-7939) and one oral vitamin K antagonist in patients with atrial fibrillation who undergo percutaneous coronary intervention (PIONEER AF-PCI). Available from: http://clinicaltrials.gov/ ct2/show/NCT01830543. NLM identifier: NCT01830543. Accessed May 17, 2013.

\section{Publish your work in this journal}

Vascular Health and Risk Management is an international, peerreviewed journal of therapeutics and risk management, focusing on concise rapid reporting of clinical studies on the processes involved in the maintenance of vascular health; the monitoring, prevention and treatment of vascular disease and its sequelae; and the involvement of metabolic disorders, particularly diabetes. This journal is indexed on PubMed Central and MedLine. The manuscript management system is completely online and includes a very quick and fair peer-review system, which is all easy to use. Visit http://www.dovepress.com/ testimonials.php to read real quotes from published authors. 